TRANSACTIONS OF THE

AMERICAN MATHEMATICAL SOCIETY

Volume 363, Number 3, March 2011, Pages 1137-1160

S 0002-9947(2010)04999-2

Article electronically published on October 19, 2010

\title{
DYNAMICS FOR THE ENERGY CRITICAL NONLINEAR WAVE EQUATION IN HIGH DIMENSIONS
}

\author{
DONG LI AND XIAOYI ZHANG
}

\begin{abstract}
T. Duyckaerts and F. Merle (2008) studied the variational structure near the ground state solution $W$ of the energy critical wave equation and classified the solutions with the threshold energy $E(W, 0)$ in dimensions $d=3,4,5$. In this paper, we extend the results to all dimensions $d \geq 6$. The main issue in high dimensions is the non-Lipschitz continuity of the nonlinearity which we get around by making full use of the decay property of $W$.
\end{abstract}

\section{INTRODUCTION}

We consider the Cauchy problem of the focusing energy critical nonlinear wave equation:

$$
\left\{\begin{array}{l}
u_{t t}-\Delta u-|u|^{\frac{4}{d-2}} u=0, \\
u(0, x)=u_{0}(x), \partial_{t} u(0, x)=u_{1}(x),
\end{array}\right.
$$

where $u(t, x)$ is a real function on $\mathbb{R} \times \mathbb{R}^{d}, d \geq 3$ and $u_{0} \in \dot{H}_{x}^{1}\left(\mathbb{R}^{d}\right), u_{1} \in L_{x}^{2}\left(\mathbb{R}^{d}\right)$. The name "energy critical" refers to the fact that the scaling

$$
u(t, x) \rightarrow u_{\lambda}(t, x)=\lambda^{-\frac{d-2}{2}} u\left(\lambda^{-1} t, \lambda^{-1} x\right)
$$

leaves both the equation and the energy invariant. Here, the energy is defined by

$$
E\left(u(t), \partial_{t} u(t)\right)=\frac{1}{2}\left\|\partial_{t} u(t)\right\|_{2}^{2}+\frac{1}{2}\|\nabla u(t)\|_{2}^{2}-\frac{d-2}{2 d}\|u(t)\|_{\frac{2 d}{d-2}}^{\frac{2 d}{d-2}}
$$

and is conserved in time.

From the classical local theory (cf. [5, 9, 16, 18, 19, 20]), for any $\left(u_{0}, u_{1}\right) \in$ $\dot{H}_{x}^{1} \times L_{x}^{2}$, there exists a unique maximal-lifespan solution of (1.1) on a time interval $\left(-T_{-}, T_{+}\right)$such that the local scattering size

$$
S_{I}(u):=\|u\|_{L_{t, x}^{\frac{2(d+1)}{d-2}}\left(I \times \mathbb{R}^{d}\right)}<\infty,
$$

for any compact interval $I \subset\left(-T_{-}, T_{+}\right)$. If $S_{\left[0, T_{+}\right)}(u)=\infty$, we say $u$ blows up forward in time. Likewise $u$ blows up backward in time if $S_{\left(-T_{+}, 0\right]}(u)=\infty$. We

Received by the editors June 16, 2008.

2010 Mathematics Subject Classification. Primary 35Q55, 35L05, 35L71.

Key words and phrases. Energy-critical wave equation, dynamical behavior, ground state.

The first and second authors were supported in part by the National Science Foundation under agreement No. DMS-0635607 and a start-up funding from the Mathematics Department of the University of Iowa. The second author was also supported by NSF grant No. 10601060 and project 973 in China.

(C)2010 American Mathematical Society Reverts to public domain 28 years from publication 
also recall the fact that the nonblowup of $u$ in one direction implies scattering in the space $\dot{H}_{x}^{1} \times L_{x}^{2}$ in that direction.

For the defocusing energy critical NLW, the global wellposedness and scattering for all finite energy solutions was established in [7, 8, 18, 17, 19, 11]. In the focusing case, depending on the size of the kinetic energy of the initial data, both scattering and blowup may occur. The threshold between blowup and scattering is believed to be determined by the ground state solution of the equation (1.1):

$$
W(x)=\left(1+\frac{|x|^{2}}{d(d-2)}\right)^{-\frac{d-2}{2}},
$$

which solves the static nonlinear wave equation

$$
\Delta W+W^{\frac{d+2}{d-2}}=0 .
$$

This was verified by Kenig-Merle [11] in dimensions $d=3,4,5$.

Theorem 1.1 (Global wellposedness and scattering [1]). Let $d=3,4,5$ and $\left(u_{0}, u_{1}\right) \in \dot{H}_{x}^{1} \times L_{x}^{2}$. Assume that $E\left(u_{0}, u_{1}\right)<E(W, 0)$. Let $u=u(t, x)$ be the maximal-lifespan solution of (1.1) on $I \times \mathbb{R}^{d}$.

i) If $\left\|\nabla u_{0}\right\|_{2}<\|\nabla W\|_{2}$, then $I=\mathbb{R}$ and the scattering size of $u$ is finite,

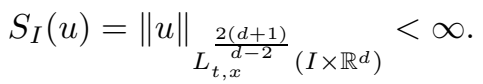

ii) If $\left\|\nabla u_{0}\right\|_{2}>\|\nabla W\|_{2}$, then $u$ blows up at finite time in both time directions, i.e., $|I|<\infty$.

Remark 1.2. In 11, Theorem 1.1 was proved in dimensions $d=3,4,5$. To generalize it to higher dimensions, one needs a stronger stability result which has been worked out for the energy critical NLS 13. After some changes, the stronger stability theory for NLS still holds for NLW, and we will address this problem elsewhere [2]. We stress that Theorem 1.4 below depends crucially on this generalization.

Theorem 1.1 confirmed that the threshold between blowup and scattering is given by the ground state $W$. Our purpose in this paper is not to investigate the global wellposedness and scattering theory below the threshold. Instead, we aim to continue the study in [4] on what will happen if the solution has the threshold energy $E(W, 0)$. In that paper, T. Duyckaerts and F. Merle carried out a very detailed study of the dynamical structure around the ground state solution $W$. They were able to give the characterization of solutions with the threshold energy in dimensions $d=3,4,5$.

In this paper, we aim to extend the results in 4 to all dimensions $d \geq 6$. Although the whole framework designed for low dimensions can also be used for the high dimensional setting, there are a couple of places where the arguments break down in high dimensions. Roughly speaking, this was mainly caused by the fact that the nonlinearity is no longer Lipschitz continuous or superlinear in Strichartz space $\dot{S}^{1}$ (see Section 2 for the definition). In the whole proof, there are mainly two places where the Lipschitz continuity and superlinearity are heavily needed. The first is in the construction of threshold solutions $W^{ \pm}$using a perturbed equation where Lipschitz continuity is used for a contraction argument. The second is in showing rigidity properties of $W^{ \pm}$where we need to show that the nonlinearity is superlinear with respect to perturbations. The superlinearity is a crucial property needed for a bootstrap argument, which was used to show that solutions exponentially close 
to $W^{ \pm}$must coincide with $W^{ \pm}$up to symmetries. To get around this problem, we will employ a similar technique which we used for the corresponding problem of energy critical NLS [15]. When constructing the threshold solution, instead of using the standard Strichartz space, we will use the weighted Sobolev space $H^{m, m}$ (see the next section for the definition). The weighted space $H^{m, m}$ turns out to be a natural space in which the nonlinearity of the perturbed equation can be proved to be Lipschitz continuous. To show the rigidity of the threshold solutions, we will show the perturbed nonlinearity of the form $R\left(v+w^{a}\right)-R\left(w^{a}\right) \sqrt{1}$ has better decay than the perturbation $v$ which already has certain exponential decay. By proving that the difference $w^{a}$ is in $H^{m, m}$, we are able to transform the perturbed nonlinearity into a form which can be treated by using the dyadic decomposition trick from [4]. The rigidity of the threshold solutions then follows after several bootstrap steps.

In all, the material in this paper allows us to extend the argument in 4] to all dimensions $d \geq 6$. This is the following

Theorem 1.3. Let $d \geq 6$. There exists a spherically symmetric solution $W^{ \pm}$ of (1.1) defined on the maximal lifespan $\left(-T_{-}\left(W^{ \pm}\right), T_{+}\left(W^{ \pm}\right)\right)$with initial data $\left(W_{0}^{ \pm}, W_{1}^{ \pm}\right) \in \dot{H}_{x}^{1} \times L_{x}^{2}$ such that

$$
\begin{array}{r}
E(W, 0)=E\left(W_{0}^{+}, W_{1}^{+}\right)=E\left(W_{0}^{-}, W_{1}^{-}\right), \\
T_{+}\left(W^{-}\right)=T_{+}\left(W^{+}\right)=+\infty, \quad \text { and } \lim _{t \rightarrow+\infty}\left\|W^{ \pm}(t)-W\right\|_{\dot{H}_{x}^{1}}=0 \\
\left\|\nabla W^{-}\right\|_{2}<\|\nabla W\|_{2}, T_{-}\left(W^{-}\right)=+\infty, S_{(-\infty, 0)}\left(W^{-}\right)<\infty \\
\left\|\nabla W^{+}\right\|_{2}>\|\nabla W\|_{2}, T_{-}\left(W^{+}\right)<\infty .
\end{array}
$$

Now we classify the solutions with the threshold energy. Since the equation is invariant under several symmetries, we can determine the solution only modulo these symmetries. Let $u(t, x), v(t, x)$ be two spacetime functions. When we say $u=v$ up to symmetries of the equation, we mean there exist $t_{0} \in \mathbb{R}, x_{0} \in \mathbb{R}^{d}$, $\lambda_{0}>0, c_{0}, c_{1} \in\{+1,-1\}$ such that

$$
u(t, x)=\frac{c_{0}}{\lambda_{0}^{\frac{d-2}{2}}} v\left(\frac{t_{0}+c_{1} t}{\lambda_{0}}, \frac{x+x_{0}}{\lambda_{0}}\right) .
$$

With this convention we have

Theorem 1.4 (Dynamical classification at the critical level). Let $d \geq 6$. Let $\left(u_{0}, u_{1}\right) \in \dot{H}_{x}^{1} \times L_{x}^{2}$ such that

$$
E\left(u_{0}, u_{1}\right)=E(W, 0) .
$$

Let $u$ be the solution of (1.1) with initial condition $\left(u_{0}, u_{1}\right)$ and let $I$ be its maximal lifespan. Then we have the following.

(a) If $\left\|\nabla u_{0}\right\|_{2}<\|\nabla W\|_{2}$, then $I=\mathbb{R}$. Moreover, either $u=W^{-}$up to symmetries of the equation, or $S_{\mathbb{R}}(u)<\infty$.

(b) If $\left\|\nabla u_{0}\right\|_{2}=\|\nabla W\|_{2}$, then $u=W$ up to symmetries of the equation.

(c) If $\left\|\nabla u_{0}\right\|_{2}>\|\nabla W\|_{2}$ and $u_{0} \in L^{2}$, then $u=W^{+}$up to symmetries of the equation or $I$ is finite.

${ }^{1}$ Here $w^{a}$ is the difference between the threshold solution and the ground state $W$; see Lemma 
The proof of Theorems 1.3 and 1.4 will follow roughly the same strategy as in [4. Here we make a remark about the proof of Theorem 1.4 The second point (b) is a direct application of the variational characterization of $W$. It only remains to prove (a) and (c). In [4, a large portion of the work was devoted to showing the exponential convergence of the solution to $W$, which after several minor changes, also works for higher dimensions. For this reason, we do not repeat that part of the argument and build our starting point on the following.

Proposition 1.5 (Exponential convergence to $W$ [4]). Let $u$ be the solution to (1.1) with initial condition $\left(u_{0}, u_{1}\right) \in \dot{H}_{x}^{1} \times L_{x}^{2}$ satisfying $E\left(u_{0}, u_{1}\right)=E(W, 0)$.

(a) In the case $\left\|\nabla u_{0}\right\|_{2}<\|\nabla W\|_{2}$, u exists globally. Suppose also that $S_{(0, \infty)}(u)$ $=\infty$. Then $S_{(-\infty, 0)}(u)<\infty$ and there exist $\lambda_{0}>0, x_{0} \in \mathbb{R}^{d}, c>0$ and $C>0$ such that

$$
\left\|\nabla\left(u(t)-\lambda^{-\frac{d-2}{2}} W\left(\lambda_{0}^{-1}\left(x+x_{0}\right)\right)\right)\right\|_{2}+\left\|\partial_{t} u(t)\right\|_{2} \leq C e^{-c t} .
$$

(b) In the case $\left\|\nabla u_{0}\right\|_{2}>\|\nabla W\|_{2}$, we also assume that $u_{0} \in L_{x}^{2}$ and that $u$ exists globally forward in time. Then there exist $c, C>0$ and $\lambda_{0}, x_{0}$ such that

$$
\left\|\nabla\left(u(t)-\lambda_{0}^{-\frac{d-2}{2}} W\left(\lambda_{0}^{-1}\left(x+x_{0}\right)\right)\right)\right\|_{2}+\left\|\partial_{t} u(t)\right\|_{2} \leq C e^{-c t} .
$$

This paper is organized as follows. In Section 2, we introduce some notation and collect some basic estimates. Section 3 is devoted to proving Theorem 1.3. In Section 4, we prove two useful estimates. In Section 5, we use the two estimates to finish the proof of Theorem 1.4 by assuming Proposition 1.5 .

\section{Preliminaries}

We use $X \lesssim Y$ or $Y \gtrsim X$ whenever $X \leq C Y$ for some constant $C>0$. We use $O(Y)$ to denote any quantity $X$ such that $|X| \lesssim Y$. We use the notation $X \sim Y$ whenever $X \lesssim Y \lesssim X$. We will add subscripts to $C$ to indicate the dependence of $C$ on the parameters. For example, $C_{i, j}$ means that the constant $C$ depends on $i, j$. The dependence of $C$ upon dimension will be suppressed.

We use the 'Japanese bracket' convention $\langle x\rangle:=\left(1+|x|^{2}\right)^{1 / 2}$.

Throughout this paper, we will use $p_{c}$ to denote the total power of nonlinearity:

$$
p_{c}=\frac{d+2}{d-2} .
$$

We write $L_{t}^{q} L_{x}^{r}$ to denote the Banach space with norm

$$
\|u\|_{L_{t}^{q} L_{x}^{r}\left(\mathbb{R} \times \mathbb{R}^{d}\right)}:=\left(\int_{\mathbb{R}}\left(\int_{\mathbb{R}^{d}}|u(t, x)|^{r} d x\right)^{q / r} d t\right)^{1 / q},
$$

with the usual modifications when either $q$ or $r$ is equal to infinity, or when the domain $\mathbb{R} \times \mathbb{R}^{d}$ is replaced by a smaller region of spacetime such as $I \times \mathbb{R}^{d}$. When $q=r$ we abbreviate $L_{t}^{q} L_{x}^{q}$ as $L_{t, x}^{q}$.

For any $s \in \mathbb{R}$, we define the fractional derivative $|\nabla|^{s}$ via the Fourier transform

$$
\widehat{|\nabla|^{s} f}(\xi)=\left(4 \pi^{2}|\xi|^{2}\right)^{\frac{s}{2}} \hat{f}(\xi)
$$

and define $\dot{W}_{x}^{s, p}\left(\mathbb{R}^{d}\right)$ to be Sobolev space with the norm

$$
\|f\|_{\dot{W}_{x}^{s, p}}=\left\||\nabla|^{s} f\right\|_{p} .
$$

When $p=2$, we write $\dot{W}_{x}^{s, 2}$ as $\dot{H}_{x}^{s}$. 
2.1. Fractional chain rule. We record the following results from [12].

Lemma 2.1 (Fractional chain rule). Let $p_{1}, p_{2}, p_{3}, p_{4} \in(1, \infty), p_{1} \in(1, \infty]$, be such that $\frac{1}{p_{1}}+\frac{1}{p_{2}}=\frac{1}{p}, \frac{1}{p_{3}}+\frac{1}{p_{4}}=\frac{1}{p}$. Let $F \in C^{1}(\mathbb{R})$ be such that $F(0)=0$. Then we have

$$
\begin{array}{r}
\left\||\nabla|^{\frac{1}{2}}(f g)\right\|_{p} \lesssim\|f\|_{p_{1}}\left\||\nabla|^{\frac{1}{2}} g\right\|_{p_{2}}+\left\||\nabla|^{\frac{1}{2}} f\right\|_{p_{3}}\|g\|_{p_{4}}, \\
\left\||\nabla|^{\frac{1}{2}} F(f)\right\|_{p} \lesssim\left\|F^{\prime}(f)\right\|_{p_{1}}\left\||\nabla|^{\frac{1}{2}} f\right\|_{p_{2}} .
\end{array}
$$

\subsection{Linear wave equation and Strichartz estimates.}

Definition 2.2 (Wave-admissible pair). Let $d \geq 6$. We say the couple $(q, r)$ is admissible if $2 \leq q \leq \infty$ and

$$
\frac{2}{q}=(d-1)\left(\frac{1}{2}-\frac{1}{r}\right)
$$

Denote $I$ to be a time slab and $\beta(r)=\frac{d+1}{2}\left(\frac{1}{2}-\frac{1}{r}\right)$. We define $\dot{S}^{1}(I), \dot{N}^{1}(I)$ to be the Banach spaces with the norms

$$
\begin{aligned}
\|u\|_{\dot{S}^{1}(I)} & =\sup _{(q, r) \text { admissible }}\left(\|u\|_{L_{t}^{q} \dot{W}_{x}^{1-\beta(r), r}\left(I \times \mathbb{R}^{d}\right)}+\left\|\partial_{t} u\right\|_{L_{t}^{q} \dot{W}_{x}^{-\beta(r), r}\left(I \times \mathbb{R}^{d}\right)}\right), \\
\|u\|_{\dot{N}^{1}(I)} & =\inf _{u=u_{1}+u_{2}}\left\|u_{1}\right\|_{L_{t}^{1} L_{x}^{2}\left(I \times \mathbb{R}^{d}\right)}+\left\||\nabla|^{\frac{1}{2}} u_{2}\right\|_{L_{t, x}^{\frac{2(d+1)}{d+3}}\left(I \times \mathbb{R}^{d}\right)} .
\end{aligned}
$$

With this notation, we write the Strichartz inequalities for the linear wave equation as follows.

Lemma 2.3 (Strichartz estimate [6, 16, 10]). Let $f \in \dot{H}_{x}^{1}, g \in L_{x}^{2}$. Let I be a time slab containing $t_{0}$. Let $F \in \dot{N}^{1}(I)$. Then the solution $u(t, x)$ to the equation

$$
\left\{\begin{array}{l}
u_{t t}-\Delta u=F, \\
u\left(t_{0}, x\right)=f(x), u_{t}\left(t_{0}, x\right)=g(x)
\end{array}\right.
$$

satisfies Duhamel's formula:

$$
u(t, x)=\cos \left(\sqrt{-\Delta}\left(t-t_{0}\right)\right) f+\frac{\sin \left(\left(t-t_{0}\right) \sqrt{-\Delta}\right)}{\sqrt{-\Delta}} g+\int_{t_{0}}^{t} \frac{\sin ((t-s) \sqrt{-\Delta})}{\sqrt{-\Delta}} F(s) d s .
$$

Moreover,

$$
\|u\|_{\dot{S}^{1}(I)} \lesssim\|f\|_{\dot{H}_{x}^{1}}+\|g\|_{2}+\|F\|_{\dot{N}^{1}(I)} .
$$

Remark 2.4. Let $d \geq 6$ and $(q, r)$ be admissible. Let $\tilde{r}$ be such that

$$
\frac{1}{q}+\frac{d}{\tilde{r}}=\frac{d-2}{2}
$$

Then by Sobolev embedding we have

$$
\|f\|_{L_{x}^{\tilde{r}}} \lesssim\|f\|_{\dot{W}_{x}^{1-\beta(r), r}}
$$

Therefore

$$
\|u\|_{L_{t}^{q} L_{x}^{\tilde{r}\left(I \times \mathbb{R}^{d}\right)}} \lesssim\|u\|_{\dot{S}^{1}(I)} .
$$

For example, we can take $(q, \tilde{r})=\left(\infty, \frac{2 d}{d-2}\right)$. Other examples will be used in this paper without explicitly mentioning this embedding. 
2.3. Space $H^{m, m}$ and its basic properties. Let $m>0$ be an integer. Define $H^{m, m}$ to be the Banach space with the norm

$$
\|f\|_{H^{m, m}}=\sum_{0 \leq j \leq m}\left\|\langle x\rangle^{m-j} \nabla^{j} f\right\|_{2} .
$$

We collect several useful lemmas.

Lemma 2.5 (Estimates of linear solutions). Let $m$ be a positive integer. Let $u$ be the solution of

$$
\left\{\begin{array}{l}
u_{t t}-\Delta u=0, \\
u(0, x)=f(x), u_{t}(0, x)=g(x) .
\end{array}\right.
$$

Then there exists an m-dependent constant $C>0$ such that

$$
\begin{aligned}
\left\|u_{t}(t)\right\|_{H^{m, m}}+\|\nabla u(t)\|_{H^{m, m}} & \leq\left(\|\nabla f\|_{H^{m, m}}+\|g\|_{H^{m, m}}\right) e^{C t}, \\
\|u(t)\|_{H^{m, m}} & \leq\left(\|\nabla f\|_{H^{m, m}}+\|g\|_{H^{m, m}}+\|f\|_{H^{m, m}}\right) e^{C t} .
\end{aligned}
$$

Proof. (2.6) follows directly from the standard energy method. The second inequality (2.7) is a consequence of (2.6) and the Fundamental Theorem of Calculus.

Lemma 2.6. Let $t_{0}>0, \alpha>0$. Let $\Sigma_{t_{0}}$ be the Banach space with the norm

$$
\|u\|_{\Sigma_{t_{0}}}=\sup _{t \geq t_{0}} e^{\alpha t}\|u(t)\|_{H^{m, m}} .
$$

Then there exists an $m$-dependent constant $C$ such that

$$
\left\|\int_{t}^{\infty} \frac{\sin ((t-\tau) \sqrt{-\Delta})}{\sqrt{-\Delta}} F(\tau) d \tau\right\|_{\Sigma_{t_{0}}} \leq \frac{1}{\alpha-C}\|F\|_{\Sigma_{t_{0}}} .
$$

Proof. Using Duhamel's formula, Lemma 2.5 gives that

$$
\left\|\frac{\sin (t \sqrt{-\Delta})}{\sqrt{-\Delta}} g\right\|_{H^{m, m}} \leq e^{C|t|}\|g\|_{H^{m, m}} .
$$

Applying this and the Minkowski inequality we have

$$
\begin{aligned}
\left\|\int_{t}^{\infty} \frac{\sin ((t-\tau) \sqrt{-\Delta})}{\sqrt{-\Delta}} F(\tau) d \tau\right\|_{H^{m, m}} & \leq \int_{t}^{\infty}\left\|\frac{\sin ((t-\tau) \sqrt{-\Delta})}{\sqrt{-\Delta}} F(\tau)\right\|_{H^{m, m}} d \tau \\
& \leq \int_{t}^{\infty} e^{C|t-\tau|}\|F(\tau)\|_{H^{m, m}} d \tau \\
& \leq \int_{t}^{\infty} e^{C(\tau-t)} e^{-\alpha \tau}\|F\|_{\Sigma_{t_{0}}} d \tau \\
& \leq \frac{e^{-\alpha t}}{\alpha-C}\|F\|_{\Sigma_{t_{0}}}
\end{aligned}
$$

which immediately gives (2.8).

We record several useful lemmas from [15. The proof can be found in [15].

Lemma 2.7 (Embedding in $\left.H^{m, m}\right)$. Let $k_{1}, k_{2}$ be nonnegative integers. Then for any $m \geq k_{1}+k_{2}+\frac{d}{2}+1$, we have

$$
\left\|\langle x\rangle^{k_{1}} \nabla^{k_{2}} f\right\|_{\infty} \lesssim\|f\|_{H^{m, m}}
$$

where the implicit constant depends only on $k_{1}, k_{2}$. 
Lemma 2.8 (Bilinear estimate in $H^{m, m}$ ). We have

$$
\|f g\|_{H^{m, m}} \lesssim\|f\|_{W^{m, \infty}}\|g\|_{H^{m, m}},
$$

with the implicit constant depending only on $\mathrm{m}$.

Lemma 2.9. Let $C>0, j \geq 2$ and $m \geq \frac{d}{2}+1+\frac{C j}{j-1}$. Then

$$
\left\|\langle x\rangle^{C j} h^{j}\right\|_{H^{m, m}} \lesssim j^{m}\|h\|_{H^{m, m}}^{j},
$$

where the implicit constant depends only on $m$.

2.4. Derivation of the perturbation equation near $W$. Let $u$ be the solution to the equation in (1.1). Let $v=u-W$. Then $v$ satisfies the equation

$$
v_{t t}-\Delta v=|v+W|^{p_{c}-1}(v+W)-W^{p_{c}} .
$$

Let $R(v)=|v+W|^{p_{c}-1}(v+W)-p_{c} W^{p_{c}-1} v-W^{p_{c}}$ and $\mathcal{L} v=\left(-\Delta-p_{c} W^{p_{c}-1}\right) v$. Then (2.10) can be written equivalently as

$$
\begin{aligned}
& v_{t t}-\Delta v=R(v)+p_{c} W^{p_{c}-1} v, \\
& v_{t t}+\mathcal{L} v=R(v) .
\end{aligned}
$$

We record the following spectral properties of $\mathcal{L}$ from [4.

Lemma 2.10 (Spectral property). The operator $\mathcal{L}$ has no positive eigenvalue and a unique negative eigenvalue $-e_{0}^{2}$ with the corresponding eigenfunction $\mathcal{Y} \in \mathcal{S}\left(\mathbb{R}^{d}\right)$, i.e.

$$
\mathcal{L} \mathcal{Y}=-e_{0}^{2} \mathcal{Y}
$$

For convenience of notation, we will assume $e_{0}>0$.

\section{The EXistence of $W^{-}, W^{+}$}

As in [4, the threshold solutions $W^{-}, W^{+}$are constructed as the limit of a sequence of near solutions $W_{k}^{a}(t, x)$. On the other hand, the asymptotic behaviors of $W^{-}$and $W^{+}$are quite different in the negative time direction (see Remark 3.5). We need the following result:

Lemma 3.1 (4]). Let $a \in \mathbb{R}$ and let $e_{0}>0$ be the same as in Lemma 2.10, There exist functions $\left\{\Phi_{j}^{a}\right\}_{j \geq 1}$ in $\mathcal{S}\left(\mathbb{R}^{d}\right)$ such that $\Phi_{1}^{a}=a \mathcal{Y}$ (see Lemma 2.10 for the definition of $\mathcal{Y})$ and if

$$
W_{k}^{a}(t, x)=W(x)+\sum_{j=1}^{k} e^{-j e_{0} t} \Phi_{j}^{a}(x),
$$

then as $t \rightarrow \infty$,

$$
\varepsilon_{k}^{a}:=\left(\partial_{t t}-\Delta\right) W_{k}^{a}-\left|W_{k}^{a}\right|^{p_{c}-1} W_{k}^{a}=O\left(e^{-(k+1) e_{0} t}\right), \text { in } \mathcal{S}\left(\mathbb{R}^{d}\right) .
$$

More precisely, $\forall J, M \geq 0, J, M$ are integers, there exists a constant $C_{J, M}$ such that

$$
\langle x\rangle^{M}\left|\nabla^{J} \varepsilon_{k}^{a}(t, x)\right| \leq C_{J, M} e^{-(k+1) e_{0} t},
$$

for all $t$ sufficiently large. 
Remark 3.2. Since all $\Phi_{j}$ are Schwartz functions, we have the following properties for the difference:

$$
v_{k}=W_{k}^{a}-W=\sum_{j=1}^{k} e^{-j e_{0} t} \Phi_{j}^{a}(x) .
$$

For any $j, l \geq 0$ and $1 \leq p \leq \infty$, there exists $C_{k, j, l}>0$ such that

$$
\begin{aligned}
\left|\langle x\rangle^{j} \nabla^{l} v_{k}(t, x)\right| & \leq C_{k, j, l} e^{-e_{0} t}, \\
\left\|\langle\cdot\rangle^{j} \nabla^{l} v_{k}(t)\right\|_{p} & \leq C_{k, j, l} e^{-e_{0} t} .
\end{aligned}
$$

Next we show that there exists a unique genuine solution $W^{a}(t, x)$ of (1.1) which can be approximated by the above constructed near solutions $W_{k}^{a}(t, x)$. The existence and uniqueness of the solution $W^{a}$ is transformed to that of $h:=W^{a}-W_{k}^{a}$ which satisfies the equation

$$
\partial_{t t} h-\Delta h=p_{c} W^{p_{c}-1} h+R\left(h+v_{k}\right)-R\left(v_{k}\right)-\varepsilon_{k}^{a} .
$$

As for the Schrödinger equation [15, we will construct the solution to (3.5) by using a fixed-point argument in the weighted Sobolev space $H^{m, m}$. The reason is that the nonlinearity in (3.5) can be shown to be Lipschitz continuous while the space used in [4] does not work for higher dimensions.

Proposition 3.3. Let $a \in \mathbb{R}$. Let $\mathcal{Y}$ and $W_{k}^{a}=W_{k}^{a}(t, x)$ be the same as in Lemma 3.1. Assume $m \geq 3 d$ is fixed. Then there exists $k_{0}>0$ and a unique solution $W^{a}(t, x)$ for the equation in (1.1) which satisfies the following: for any $k \geq k_{0}$, there exists $t_{k} \geq 0$ such that $\forall t \geq t_{k}$,

$$
\left\|W^{a}(t)-W_{k}^{a}(t)\right\|_{H^{m, m}} \leq e^{-\left(k+\frac{1}{2}\right) e_{0} t} .
$$

Moreover, we have

$$
\left\|\nabla\left(W^{a}(t)-W_{k}^{a}\right)\right\|_{H^{m, m}}+\| \partial_{t}\left(W^{a}(t)-W_{k}^{a}(t) \|_{H^{m, m}} \leq e^{-\left(k+\frac{1}{2}\right) e_{0} t} .\right.
$$

Proof. Let $h=W^{a}-W_{k}^{a}$. Then $W^{a}$ is the solution of (1.1) as long as $h$ is a solution of the equation (3.5) . By Duhamel's formula, the existence of the solution to (3.5) which satisfies the decay conditions (3.6), (3.7) is transformed into the existence of the solution to the following integral equation for large time $t$ :

$$
\begin{aligned}
h(t) & =-\int_{t}^{\infty} \frac{\sin ((t-\tau) \sqrt{-\Delta})}{\sqrt{-\Delta}}\left(p_{c} W^{p_{c}-1} h+R\left(h+v_{k}\right)-R\left(v_{k}\right)-\varepsilon_{k}^{a}\right)(\tau) d \tau \\
& :=\Phi(h)(t) .
\end{aligned}
$$

Define the space $\Sigma_{t_{k}}$ to be the space with the norm

$$
\|f\|_{\Sigma_{t_{k}}}=\sup _{t \geq t_{k}} e^{\alpha t}\|f(t)\|_{H^{m, m}}, \alpha=\left(k+\frac{1}{2}\right) e_{0},
$$

and the unit ball

$$
B_{k}=\left\{f=f(t, x) ;\|f\|_{\Sigma_{t_{k}}} \leq 1\right\}
$$


We will show that $\Phi$ is a contraction on $B_{k}$. Taking $h \in B_{k}$, we compute the $H^{m, m}$ norm of $\Phi(h)(t)$ as follows:

$$
\begin{aligned}
\|\Phi(h)(t)\|_{H^{m, m}} & \leq p_{c} \int_{t}^{\infty}\left\|\frac{\sin ((t-\tau) \sqrt{-\Delta})}{\sqrt{-\Delta}} W^{p_{c}-1} h(\tau)\right\|_{H^{m, m}} d \tau \\
& +\int_{t}^{\infty}\left\|\frac{\sin ((t-\tau) \sqrt{-\Delta})}{\sqrt{-\Delta}}\left(R\left(h+v_{k}\right)-R\left(v_{k}\right)\right)(\tau)\right\|_{H^{m, m}} d \tau \\
& +\int_{t}^{\infty}\left\|\frac{\sin ((t-\tau) \sqrt{-\Delta})}{\sqrt{-\Delta}} \varepsilon_{k}^{a}(\tau)\right\|_{H^{m, m}} d \tau .
\end{aligned}
$$

To estimate (3.10), we use Lemma 2.5 and Lemma 2.8 to get

$$
\begin{aligned}
(3.10) & \lesssim \int_{t}^{\infty} e^{C|t-\tau|}\left\|W^{p_{c}-1} h(\tau)\right\|_{H^{m, m}} d \tau \\
& \lesssim \int_{t}^{\infty} e^{C|t-\tau|}\left\|W^{p_{c}-1}\right\|_{W^{m, \infty}}\|h(\tau)\|_{H^{m, m}} d \tau \\
& \lesssim \int_{t}^{\infty} e^{C(\tau-t)} e^{-\alpha \tau}\|h\|_{\Sigma_{t_{k}}} d \tau \\
& \lesssim e^{-C t}\|h\|_{\Sigma_{t_{k}}} \int_{t}^{\infty} e^{-(\alpha-C) \tau} d \tau \\
& \lesssim \frac{1}{\alpha-C} e^{-\alpha t}\|h\|_{\Sigma_{t_{k}}} .
\end{aligned}
$$

Since $\alpha=\left(k+\frac{1}{2}\right) e_{0}$, by taking $k_{0}$ sufficiently large, we have

$$
\text { (3.10) } \leq \frac{1}{100} e^{-\alpha t}\|h\|_{\Sigma_{t_{k}}} \leq \frac{1}{100} e^{-\alpha t}
$$

for all $k \geq k_{0}$.

Now we deal with (3.12). Note that by Lemma 3.1, $\varepsilon_{k}^{a}(t)=O\left(e^{-(k+1) e_{0} t}\right)$ in $\mathcal{S}\left(\mathbb{R}^{d}\right)$. This implies

$$
\left\|\varepsilon_{k}^{a}(t)\right\|_{H^{m, m}} \leq C_{k} e^{-(k+1) e_{0} t}
$$

Thus,

$$
\begin{aligned}
(3.12) & \leq \int_{t}^{\infty} e^{C|t-\tau|}\left\|\varepsilon_{k}^{a}(\tau)\right\|_{H^{m, m}} d \tau \\
& \leq C_{k} \int_{t}^{\infty} e^{C|t-\tau|} e^{-(k+1) e_{0} \tau} d s \tau \\
& \leq C_{k} \frac{e^{-\frac{1}{2} e_{0} t}}{(k+1) e_{0}-C} e^{-\left(k+\frac{1}{2}\right) e_{0} t} \leq \frac{1}{100} e^{-\alpha t},
\end{aligned}
$$

if $t \geq t_{k}$ and $t_{k}$ is sufficiently large.

It remains to estimate (3.11). The reason that we can take $m$ derivatives is that both $v_{k}$ and $h$ are small compared to $W$. Indeed by Remark 3.2, we have

$$
\left|v_{k}(t, x)\right|<\frac{1}{2} W(x), \quad \forall t \geq t_{k}, x \in \mathbb{R}^{d} .
$$

Moreover, since $h \in \Sigma_{t_{k}}$ and $m \geq 3 d$, by Lemma 2.7 we have

$$
\left\|\langle x\rangle^{d-2} h(t)\right\|_{\infty} \lesssim\|h(t)\|_{H^{m, m}} \leq e^{-\alpha t}\|h\|_{\Sigma_{t_{k}}} .
$$


As a consequence, we have

$$
|h(t, x)| \lesssim e^{-\alpha t}\langle x\rangle^{-(d-2)}\|h\|_{\Sigma_{t_{k}}} \leq \frac{1}{4} W(x) .
$$

Using (3.16) and (3.17) together with the expansion for the real analytic function $P(s)=|1+s|^{p_{c}-1}(1+s)$ for $|s| \leq \frac{3}{4}$, which takes the form

$$
P(s)=1+p_{c} s+\sum_{j \geq 2} a_{j} s^{j},\left|a_{j}\right| \lesssim 1
$$

we write

$$
\begin{aligned}
& R\left(v_{k}+h\right)-R\left(v_{k}\right) \\
& =W^{p_{c}}\left(\left|1+\frac{v_{k}+h}{W}\right|^{p_{c}-1}\left(1+\frac{v_{k}+h}{W}\right)-\left|1+\frac{v_{k}}{W}\right|^{p_{c}-1}\left(1+\frac{v_{k}}{W}\right)-p_{c} \frac{h}{W}\right) \\
& =\sum_{\substack{j \geq 2 \\
1 \leq i \leq j}} a_{j} C_{i, j} W^{p_{c}-j} v_{k}^{j-i} h^{i},
\end{aligned}
$$

where $C_{i, j}=\frac{j !}{i !(j-i) !} \leq 2^{j}$. By the triangle inequality and Lemma 2.8, we estimate the $H^{m, m}$-norm of (3.19) as follows:

$$
\begin{aligned}
& \left\|R\left(v_{k}+h\right)(t)-R\left(v_{k}\right)(t)\right\|_{H^{m, m}} \\
& \lesssim \sum_{\substack{j \geq 2 \\
1 \leq i \leq j}} 2^{j}\left\|W^{p_{c}-j} v_{k}(t)^{j-i} h(t)^{i}\right\|_{H^{m, m}} \\
& \lesssim \sum_{j \geq 2} 2^{j}\left\|W^{-j} v_{k}(t)^{j-1} h(t)\right\|_{H^{m, m}}+\sum_{\substack{j \leq 2 \\
2 \leq i \leq j}} 2^{j}\left\|\left(W^{-1} v_{k}(t)\right)^{j-i} W^{-i} h(t)^{i}\right\|_{H^{m, m}} \\
& \lesssim \sum_{j \geq 2} 2^{j}\left\|W^{-j} v_{k}(t)^{j-1}\right\|_{W^{m, \infty}}\|h(t)\|_{H^{m, m}} \\
& \quad+\sum_{\substack{j \geq 2 \\
2 \leq i \leq j}} 2^{j}\left\|\left(W^{-1} v_{k}(t)\right)^{j-i}\right\|_{W^{m, \infty}}\left\|W^{-i} h^{i}\right\|_{H^{m, m}} .
\end{aligned}
$$

Here we have split the sum in the index $i$ because our Lemma 2.9 requires $i \geq 2$. Now by Remark 3.2 and Lemma 2.9 we have

$$
\begin{aligned}
\left\|W^{-i} h^{i}(t)\right\|_{H^{m, m}} & \lesssim i^{m}\|h(t)\|_{H^{m, m}}^{i} \\
\left\|W^{-j} v_{k}(t)^{j-1}\right\|_{W^{m, \infty}} & \lesssim j^{m} C_{k} e^{-(j-1) e_{0} t} \\
\left\|\left(W^{-1} v_{k}(t)\right)^{j-i}\right\|_{W^{m, \infty}} & \lesssim j^{m} C_{k} e^{-(j-i) e_{0} t}
\end{aligned}
$$

Noting moreover that

$$
\|h(t)\|_{H^{m, m}} \leq e^{-\alpha t}\|h\|_{\Sigma_{t_{k}}}
$$


we have

$$
\begin{aligned}
\left\|R\left(v_{k}+h\right)(t)-R\left(v_{k}\right)(t)\right\|_{H^{m, m}} & \lesssim \sum_{\substack{j \geq 2 \\
1 \leq i \leq j}} 2^{j} j^{2 m} C_{k} e^{-(j-i) e_{0} t}\left(e^{-\alpha t}\|h\|_{\Sigma_{t_{k}}}\right)^{i} \\
& \lesssim e^{-\alpha t}\|h\|_{\Sigma_{t_{k}}} \sum_{\substack{j \geq 2 \\
1 \leq i \leq j}} 2^{j} j^{2 m} C_{k} e^{\left(-\alpha(i-1)-(j-i) e_{0}\right) t} \\
& \lesssim e^{-\alpha t}\|h\|_{\Sigma_{t_{k}}} \sum_{\substack{j \geq 2 \\
1 \leq i \leq j}} 2^{j} j^{2 m} C_{k} e^{-(j-1) e_{0} t_{k}}
\end{aligned}
$$

where in the second inequality we have dropped the term $\|h\|_{\Sigma_{t_{k}}}^{i-1}$ since $\|h\|_{\Sigma_{t_{k}}} \leq 1$. Obviously the last series can be made arbitrarily small if we choose $t_{k}$ sufficiently large. This gives us

$$
\left\|R\left(v_{k}+h\right)(t)-R\left(v_{k}\right)(t)\right\|_{H^{m, m}} \leq \frac{1}{100} e^{-\alpha t}\|h\|_{\Sigma_{t_{k}}}
$$

Applying this estimate and Lemma 2.5 we have

$$
\begin{aligned}
\text { (3.11) } & \leq \int_{t}^{\infty} e^{C|t-\tau|}\left\|R\left(h+v_{k}\right)(\tau)-R\left(v_{k}\right)(\tau)\right\|_{H^{m, m}} d \tau \\
& \leq \frac{1}{100} \int_{t}^{\infty} e^{C|t-\tau|} e^{-\alpha \tau}\|h\|_{\Sigma_{t_{k}}} d \tau \\
& \leq \frac{1}{100} e^{-\alpha t}\|h\|_{\Sigma_{t_{k}}} .
\end{aligned}
$$

Collecting the estimates (3.14), (3.15) and (3.20) we obtain

$$
\|\Phi(h)(t)\|_{H^{m, m}} \leq \frac{1}{10} e^{-\alpha t}\|h\|_{\Sigma_{t_{k}}}
$$

for all $t \geq t_{k}, k \geq k_{0}$. Therefore

$$
\|\Phi(h)(t)\|_{\Sigma_{t_{k}}} \leq \frac{1}{10}
$$

which shows that $\Phi$ maps $B_{k}$ to itself. To show that $\Phi$ is a contraction, we choose $h_{1}, h_{2} \in B_{k}$ and estimate

$$
\begin{aligned}
\| \Phi\left(h_{1}\right)(t) & -\Phi\left(h_{2}\right)(t) \|_{H^{m, m}} \\
& \leq p_{c} \int_{t}^{\infty}\left\|\frac{\sin ((t-\tau) \sqrt{-\Delta})}{\sqrt{-\Delta}} W^{p_{c}-1}\left(h_{1}-h_{2}\right)(\tau)\right\| d \tau \\
& +\int_{t}^{\infty}\left\|\frac{\sin ((t-\tau) \sqrt{-\Delta})}{\sqrt{-\Delta}}\left(R\left(h_{1}+v_{k}\right)(\tau)-R\left(h_{2}+v_{k}\right)(\tau)\right)\right\| d \tau
\end{aligned}
$$

The estimate of (3.21) is the same as 3.10). We get

$$
\text { (3.21) } \leq \frac{1}{100} e^{-\alpha t}\left\|h_{1}-h_{2}\right\|_{\Sigma_{t_{k}}}, \quad \forall k \geq k_{0} .
$$


To estimate (3.22), we use (3.18) to write

$$
\begin{aligned}
R\left(h_{1}+v_{k}\right)-R\left(h_{2}+v_{k}\right)= & W^{p_{c}}\left(\left|1+\frac{h_{1}+v_{k}}{W}\right|^{p_{c}-1}\left(1+\frac{h_{1}+v_{k}}{W}\right)\right. \\
& \left.-\left|1+\frac{h_{2}+v_{k}}{W}\right|^{p_{c}-1}\left(1+\frac{h_{2}+v_{k}}{W}\right)-p_{c} W^{p_{c}-1}\left(h_{1}-h_{2}\right)\right) \\
= & \sum_{j \geq 2} a_{j} W^{p_{c}-j}\left(\left(h_{1}+v_{k}\right)^{j}-\left(h_{2}+v_{k}\right)^{j}\right) \\
= & \sum_{\substack{j \geq 2 \\
1 \leq i \leq j}} a_{j} C_{i, j}\left(h_{2}+v_{k}\right)^{j-i}\left(h_{1}-h_{2}\right)^{i} .
\end{aligned}
$$

With minor changes, this term can be treated in the same manner as (3.19), so we have

$$
\text { (3.22) } \leq \frac{1}{100} e^{-\alpha t}\left\|h_{1}-h_{2}\right\|_{\Sigma_{t_{k}}}, \forall k \geq k_{0}, t \geq t_{k} .
$$

Therefore,

$$
\begin{aligned}
\left\|\Phi\left(h_{1}\right)(t)-\Phi\left(h_{2}\right)(t)\right\|_{H^{m, m}} & \leq \frac{1}{10} e^{-\alpha t}\left\|h_{1}-h_{2}\right\|_{\Sigma_{t_{k}}}, \quad \forall k \geq k_{0}, t \geq t_{k}, \\
\left\|\Phi\left(h_{1}\right)-\Phi\left(h_{2}\right)\right\|_{\Sigma_{t_{k}}} & \leq \frac{1}{10}\left\|h_{1}-h_{2}\right\|_{\Sigma_{t_{k}}} .
\end{aligned}
$$

This proves the map $\Phi$ is a contraction on $B_{k}$; hence there exists a unique solution $h$ to the equation (3.5) such that

$$
\|h(t)\|_{H^{m, m}} \leq e^{-\alpha t}, \forall t \geq t_{k}, k \geq k_{0} .
$$

Note that $h=W^{a}-W_{k}^{a}$. This means that for any $k \geq k_{0}$, there exists a unique solution $W^{a}(t)$ to the equation (1.1) on $\left[t_{k}, \infty\right)$ such that

$$
\left\|W^{a}(t)-W_{k}^{a}(t)\right\|_{H^{m, m}} \leq e^{-\left(k+\frac{1}{2}\right) e_{0} t} .
$$

We need to show that $W^{a}(t, x)$ is independent of $k$. Indeed, let $k_{1}<k_{2}$ and $W^{a}$, $\widetilde{W^{a}}$ be the corresponding solutions such that

$$
\begin{aligned}
& \left\|W^{a}(t)-W_{k_{1}}^{a}(t)\right\|_{H^{m, m}} \leq e^{-\left(k_{1}+\frac{1}{2}\right) e_{0} t}, \quad \forall t \geq t_{k_{1}}, \\
& \left\|\widetilde{W^{a}}(t)-W_{k_{2}}^{a}(t)\right\|_{H^{m, m}} \leq e^{-\left(k_{2}+\frac{1}{2}\right) e_{0} t}, \quad \forall t \geq t_{k_{2}} .
\end{aligned}
$$

Without loss of generality we also assume $t_{k_{1}} \leq t_{k_{2}}$. Then the triangle inequality gives that

$$
\begin{aligned}
\left\|\widetilde{W^{a}}(t)-W_{k_{1}}^{a}(t)\right\|_{H^{m, m}} & \leq\left\|\widetilde{W^{a}}(t)-W_{k_{2}}^{a}(t)\right\|_{H^{m, m}}+\left\|\sum_{k_{1}<j \leq k_{2}} e^{-j e_{0} t} \Phi_{j}\right\|_{H^{m, m}} \\
& \leq e^{-\left(k_{1}+\frac{1}{2}\right) e_{0} t}, \quad \forall t \geq t_{k_{2}} .
\end{aligned}
$$

Therefore $W^{a}(t)=\widetilde{W^{a}}(t)$ on $\left[t_{k_{2}}, \infty\right)$, and we conclude $W^{a} \equiv \widetilde{W^{a}}$ by uniqueness of the solutions to (1.1). This shows that $W^{a}$ does not depend on $k$. 
We finally verify (3.7). Using Duhamel's formula (3.8) and Lemma 2.5, we have

$$
\begin{aligned}
& \left\|h_{t}(t)\right\|_{H^{m, m}}+\|\nabla h(t)\|_{H^{m, m}} \\
& \leq \int_{t}^{\infty} \| \partial_{t} \frac{\sin ((t-\tau) \sqrt{-\Delta})}{\sqrt{-\Delta}}\left(p_{c} W^{p_{c}-1} h(\tau)+R\left(h+v_{k}\right)(\tau)-R\left(v_{k}\right)(\tau)\right. \\
& +\int_{t}^{\infty}\left\|\nabla \frac{\sin ((t-\tau) \sqrt{-\Delta})}{\sqrt{-\Delta}}\left(p_{c} W^{p_{c}-1} h(\tau)\right)\right\|_{H^{m, m}} d \tau \\
& \left.\leq \int_{t}^{\infty} e^{C|t-\tau|} \| p_{c} W^{p_{c}-1} h(\tau)+R\left(h+v_{k}\right)(\tau)-R\left(v_{k}\right)(\tau) \quad-\varepsilon_{k}^{a}(\tau)\right) \|_{H^{m, m}} d \tau \\
& \leq p_{c} \int_{t}^{\infty} e^{C|t-\tau|}\left\|W^{p_{c}-1} h(\tau)\right\|_{H^{m, m}} d \tau \\
& \quad+\int_{t}^{\infty} e^{C|t-\tau|}\left\|R\left(h+v_{k}\right)(\tau)-R\left(v_{k}\right)(\tau)\right\|_{H^{m, m}} d \tau \\
& \quad+\int_{t}^{\infty} e^{C|t-\tau|}\left\|\varepsilon_{k}^{a}(\tau)\right\|_{H^{m, m}} d \tau .
\end{aligned}
$$

These terms have been estimated before (see (3.10), (3.11), (3.12)). With the condition (3.23), we have

$$
\left\|h_{t}(t)\right\|_{H^{m, m}}+\|\nabla h(t)\|_{H^{m, m}} \leq e^{-\left(k+\frac{1}{2}\right) e_{0} t} .
$$

The proposition is proved.

Corollary 3.4. Let $k_{0}$ be the same as in Proposition 3.3 , let $v_{k_{0}}=\sum_{j=1}^{k_{0}} e^{-j e_{0} t} \phi_{j}(x)$ and $w^{a}=W^{a}-W$. Then there exists $t_{0}>0$ such that for all $t \geq t_{0}$ and all $2 \leq p \leq \infty$,

$$
\begin{array}{r}
\left\|\langle x\rangle^{l_{1}} \nabla^{l_{2}}\left(w^{a}(t)-v_{k_{0}}(t)\right)\right\|_{L_{x}^{p}} \lesssim e^{-\left(k_{0}+1\right) e_{0} t} \leq e^{-\left(k_{0}+\frac{1}{2}\right) e_{0} t}, \\
\left\|\langle x\rangle^{l_{1}} \nabla^{l_{2}} w^{a}(t)\right\|_{L_{x}^{p}} \lesssim e^{-e_{0} t} \leq e^{-\frac{1}{2} e_{0} t},
\end{array}
$$

as long as $l_{1}+l_{2}+\frac{d}{2}+1 \leq m$. In particular,

$$
\left\|w^{a}-v_{k_{0}}\right\|_{\dot{S}^{1}([t, \infty))} \leq e^{-k_{0} e_{0} t}, \quad\left\|w^{a}\right\|_{\dot{S}^{1}([t, \infty))} \leq e^{-\frac{1}{2} e_{0} t} .
$$

Proof. By Proposition 3.3 ,

$$
\left\|w^{a}(t)-v_{k_{0}}(t)\right\|_{H^{m, m}}=\left\|W^{a}(t)-W_{k_{0}}^{a}(t)\right\|_{H^{m, m}} \leq e^{-\left(k_{0}+\frac{1}{2}\right) e_{0} t}, \quad \forall t \geq t_{k_{0}} .
$$

An application of Sobolev embedding (see Lemma 2.7) and interpolation yields that for any $2 \leq p \leq \infty$ and $t$ sufficiently large,

$$
\begin{aligned}
\left\|\langle x\rangle^{l_{1}} \nabla^{l_{2}}\left(w^{a}(t)-v_{k_{0}}(t)\right)\right\|_{L_{x}^{p}} & \lesssim e^{-\left(k_{0}+\frac{1}{2}\right) e_{0} t} \\
& \leq \frac{1}{2} e^{-k_{0} e_{0} t}
\end{aligned}
$$


as long as $m \geq l_{1}+l_{2}+\frac{d}{2}+1$. In particular, for any $2 \leq r \leq \frac{2(d-1)}{d-3}$,

$$
\begin{aligned}
& \left\||\nabla|^{1-\beta(r)}\left(w^{a}(t)-v_{k_{0}}(t)\right)\right\|_{L_{x}^{r}}+\||\nabla|^{-\beta(r)}\left(w^{a}(t)-v_{k_{0}}(t) \|_{L_{x}^{r}}\right. \\
\lesssim & \left\|\langle\nabla\rangle\left(w^{a}(t)-v_{k_{0}}(t)\right)\right\|_{L_{x}^{r}}+\left\|w^{a}(t)-v_{k_{0}}(t)\right\|_{L_{x}^{\frac{4}{r(d+1)+2 d-2}}} \\
\lesssim & e^{-\left(k_{0}+\frac{1}{2}\right) e_{0} t} .
\end{aligned}
$$

In the last equality, we use the fact that $\frac{4 r d}{r(d+1)+2 d-2} \geq 2$ for all $r \geq 2$. Integrating the time variable over $[t, \infty)$, we obtain

$$
\left\|w^{a}-v_{k_{0}}\right\|_{\dot{S}^{1}([t, \infty))} \lesssim e^{-\left(k_{0}+\frac{1}{2}\right) e_{0} t} \leq \frac{1}{2} e^{-k_{0} e_{0} t} .
$$

On the other hand, since $v_{k_{0}}$ is the combination of Schwartz functions, we have by (3.4),

$$
\left\|v_{k_{0}}\right\|_{\dot{S}^{1}([t, \infty))} \lesssim e^{-e_{0} t} \leq \frac{1}{2} e^{-\frac{e_{0}}{2} t},
$$

for all $t$ sufficiently large. The estimates of $w^{a}$ then follow from the triangle inequality.

Remark 3.5. From the construction of $W^{ \pm}(t)$, it is clear that they both approach the ground state $W$ exponentially fast as $t \rightarrow+\infty$. For the behavior of $W^{ \pm}$in the negative time direction, we can apply the same argument in [3] (see the proof of Theorem 1, Proposition 2.8, Proposition 3.1 and Subsection 6.4 for instance) to conclude that $W^{-}$scatters when $t \rightarrow-\infty$ and $W^{+}$blows up at finite time.

\section{Two useful estimates}

First we show that $R(v)$ is superlinear in $v$. We have

Lemma 4.1 (Superlinearity of $R(v)$ ). Let I be a time slab. We have

$$
\begin{aligned}
\|R(v)(t)\|_{L_{x}^{\frac{2 d}{d+2}}} & \lesssim\|v(t)\|_{\dot{H}_{x}^{1}}^{p_{c}}, \\
\|R(v)\|_{\dot{N}^{1}(I)} & \lesssim\|v\|_{\dot{S}^{1}(I)}^{\frac{d+3}{d+1}}+\|v\|_{\dot{S}^{1}(I)}^{p_{c}} .
\end{aligned}
$$

Proof. By the definition of $R(v)$, we write

$$
\begin{aligned}
R(v) & =|v+W|^{p_{c}-1}(v+W)-p_{c} W^{p_{c}-1} v-W^{p_{c}}-|v|^{p_{c}-1} v+|v|^{p_{c}-1} v \\
& =W^{p_{c}} J\left(\frac{v}{W}\right)+|v|^{p_{c}-1} v,
\end{aligned}
$$

where

$$
J(s)=|1+s|^{p_{c}-1}(1+s)-p_{c} s-1-|s|^{p_{c}-1} s .
$$

Note that $J(s)$ is differentiable and for $d \geq 6$,

$$
J(s) \leq\left\{\begin{array}{ll}
|s|, & |s| \geq \frac{1}{2}, \\
|s|^{p_{c}}, & |s|<\frac{1}{2},
\end{array} \quad J^{\prime}(s) \leq \begin{cases}1, & |s|^{p_{c}-1} \geq \frac{1}{2} \\
|s|, & |s|<\frac{1}{2} .\end{cases}\right.
$$

We first give a quick proof of (4.1). Since by (4.4), $J(s) \lesssim|s|^{p_{c}}$, we have

$$
\begin{aligned}
\|R(v)(t)\|_{L_{x}^{\frac{2 d}{d+2}}} & \lesssim\left\|\left.v(t)\right|^{p_{c}}\right\|_{L_{x}^{\frac{2 d}{d+2}}} \\
& \lesssim\|v(t)\|_{L_{x}^{\frac{2 d}{d-2}}}^{p_{c}} \lesssim\|v(t)\|_{H_{x}^{1}}^{p_{c}} .
\end{aligned}
$$


Now we compute the $\dot{N}^{1}$ norm of $R(v)$. In the following, all spacetime norms are on $I \times \mathbb{R}^{d}$. We have

$$
\|R(v)\|_{\dot{N}^{1}(I)} \leq\left\||\nabla|^{\frac{1}{2}}\left(W^{p_{c}} J\left(\frac{v}{W}\right)\right)\right\|_{L_{t, x}^{\frac{2(d+1)}{d+3}}}+\left\||\nabla|^{\frac{1}{2}}\left(|v|^{p_{c}-1} v\right)\right\|_{L_{t, x}^{\frac{2(d+1)}{d+3}}} .
$$

For the second term, we use Lemma 2.1 and the Hölder inequality to get

$$
\begin{aligned}
\left\||\nabla|^{\frac{1}{2}}\left(|v|^{p_{c}-1} v\right)\right\|_{L_{t, x}^{\frac{2(d+1)}{d+3}}} & \leq\left\||v|^{p_{c}-1}\right\|_{L_{t, x}^{\frac{d+1}{2}}}\left\||\nabla|^{\frac{1}{2}} v\right\|_{L_{t, x}^{\frac{2(d+1)}{d-1}}} \\
& \lesssim\|v\|_{L_{t, x}^{\frac{2(d+2)}{p^{-2}}}}^{p^{\frac{2(d)}{d}}}\left\||\nabla|^{\frac{1}{2}} v\right\|_{L_{t, x}^{\frac{2(d+1)}{d-1}}} \\
& \lesssim\|v\|_{\dot{S}^{1}(I)}^{p_{c}},
\end{aligned}
$$

which is good for us. For the first term on the RHS of (4.5), we follow the idea in 44 and cut it into dyadic pieces. To this end, we introduce a smooth cutoff function $\phi(x)$ which satisfies: $\phi(x)=1$ when $|x| \leq 1$ and $\phi(x)=0$ when $|x|>2$. Denote

$$
\psi_{k}(x)= \begin{cases}\phi(x), & k=0 \\ \phi\left(\frac{x}{2^{k}}\right)-\phi\left(\frac{x}{2^{k-1}}\right), & k \geq 1 .\end{cases}
$$

Then we verify that

$$
\begin{gathered}
\operatorname{supp} \psi_{k}(x)=\left\{x:|x| \sim 2^{k}\right\} ; \operatorname{supp} \psi_{0}(x)=\{x:|x| \lesssim 1\}, \\
\sum_{k \geq 0} \psi_{k}(x)=1, \forall x \in \mathbb{R}^{d} .
\end{gathered}
$$

We also introduce a "fat" cutoff function $\tilde{\psi}_{k}(x)$ which equals one on the support of $\psi_{k}$. By rescaling, it is straightforward to verify that: for any $1 \leq p \leq \infty$ and $\beta \in \mathbb{R}$,

$$
\left\|\langle x\rangle^{\beta} \psi_{k}\right\|_{p} \lesssim 2^{k\left(\frac{d}{p}+\beta\right)},\left\||\nabla|^{\frac{1}{2}}\left(\langle x\rangle^{\beta} \psi_{k}\right)\right\|_{p} \lesssim 2^{k\left(\frac{d}{p}+\beta-\frac{1}{2}\right)} .
$$

The same estimates hold if we replace $\psi_{k}$ by $\tilde{\psi}_{k}$.

Now we use Lemma 2.1 to estimate the first term on the RHS in (4.5) as follows:

$$
\begin{aligned}
& \left\||\nabla|^{\frac{1}{2}}\left(W^{p_{c}} J\left(\frac{v}{W}\right)\right)\right\|_{L_{t, x}^{\frac{2(d+1)}{d+3}}} \\
& \leq \sum_{k \geq 0}\left\||\nabla|^{\frac{1}{2}}\left(W^{p_{c}} \psi_{k} J\left(\tilde{\psi}_{k} \frac{v}{W}\right)\right)\right\|_{L_{t, x}^{\frac{2(d+1)}{d+3}}} \\
& \lesssim \sum_{k \geq 0}\left\||\nabla|^{\frac{1}{2}}\left(W^{p_{c}} \psi_{k}\right)\right\|_{p_{1}}\left\|J\left(\tilde{\psi}_{k} \frac{v}{W}\right)\right\|_{L_{t}^{\frac{2(d+1)}{d+3}}} L_{x}^{p_{2}} \\
& \quad+\sum_{k \geq 0}\left\|W^{p_{c}} \psi_{k}\right\|_{q_{1}}\left\||\nabla|^{\frac{1}{2}} J\left(\tilde{\psi}_{k} \frac{v}{W}\right)\right\|_{L_{t}^{\frac{2(d+1)}{d+3}}} L_{x}^{q_{2}^{2}},
\end{aligned}
$$

where $1<p_{1}, p_{2}, q_{1}, q_{2}<\infty$ are chosen such that

$$
\frac{1}{p_{1}}+\frac{1}{p_{2}}=\frac{1}{q_{1}}+\frac{1}{q_{2}}=\frac{d+3}{2(d+1)} .
$$


We first deal with (4.8). Choosing $p_{1}=\frac{4 d(d+1)}{3(d+3)}, p_{2}=\frac{4 d(d+1)}{(d+3)(2 d-3)}, p_{3}=\frac{4 d(d+1)}{3(d+3)}$, $p_{4}=\frac{2 d(d+1)}{d^{2}-9}$, we check that

$$
\frac{1}{p_{1}}+\frac{1}{p_{2}}=\frac{d+3}{2(d+1)}, \frac{1}{p_{2}}=\frac{1}{p_{3}}+\frac{1}{p_{4}} .
$$

Noting $W^{p_{c}} \lesssim\langle x\rangle^{-(d+2)},|J(s)| \lesssim|s|^{\frac{d+3}{d+1}}$ and using (4.7), we bound the summand in (4.8) by

$$
\begin{aligned}
2^{k\left(\frac{d}{p_{1}}-d-2-\frac{1}{2}\right)}\left\|\tilde{\psi}_{k} W^{-\frac{d+3}{d+1}} v^{\frac{d+3}{d+1}}\right\|{ }_{L_{t}^{\frac{2(d+1)}{d+3}} L_{x}^{p_{2}}} \\
\quad \lesssim 2^{k\left(\frac{d}{p_{1}}-d-\frac{5}{2}\right)}\left\|\tilde{\psi}_{k} W^{-\frac{d+3}{d+1}}\right\|_{L_{x}^{p_{3}}}\|v\|^{\frac{d+3}{d+1}}{ }_{L_{t}^{2} L_{x}^{\frac{p_{4}(d+3)}{d+1}}} \\
\quad \lesssim 2^{k\left(\frac{d}{p_{1}}-d-\frac{5}{2}\right)} 2^{k\left(\frac{d}{p_{3}}+\frac{(d-2)(d+3)}{d+1}\right)}\|v\|_{L_{t}^{2} \frac{d+3}{d+1}}^{\frac{2 d}{d-3}} \\
\quad \lesssim 2^{-k \frac{d+4}{d+1}\|v\|_{\dot{S}^{1}(I)}^{\frac{d+3}{d+1}}}
\end{aligned}
$$

Summing in $k$, we obtain

$$
\text { (4.8) } \lesssim\|v\|_{\dot{S}^{1}(I)}^{\frac{d+3}{d+1}} .
$$

We now deal with (4.9). Let $q_{1}=\frac{d(d+1)}{6}, q_{2}=\frac{2 d(d+1)}{d^{2}+3 d-12}, q_{3}=\frac{2(d+1)}{d-1}, q_{4}=$ $\frac{d(d+1)}{2(d-3)}$. We verify that

$$
\frac{1}{q_{1}}+\frac{1}{q_{2}}=\frac{d+3}{2(d+1)}, \frac{1}{q_{3}}+\frac{1}{q_{4}}=\frac{1}{q_{2}} .
$$

Note by (4.4), we have $\left|J^{\prime}(s)\right| \lesssim|s|^{\frac{4}{d+1}}$. Using (4.7) and Lemma 2.1 we estimate the summand in (4.9) by

$$
\begin{aligned}
& \left\|W^{p_{c}} \psi_{k}\right\|_{L_{x}^{q_{1}}}\left\||\nabla|^{\frac{1}{2}} J\left(\tilde{\psi}_{k} W^{-1} v\right)\right\|_{L_{t}^{\frac{2(d+1)}{d+3}}} L_{x}^{q_{2}}
\end{aligned}
$$

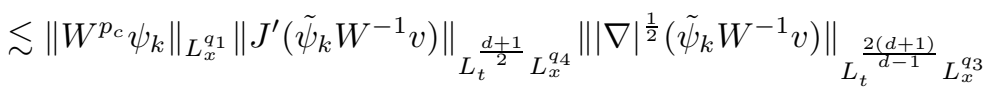

$$
\begin{aligned}
& \lesssim\left\|W^{p_{c}} \psi_{k}\right\|_{L_{x}^{q_{1}}\left\|\tilde{\psi}_{k} W^{-1} v\right\|_{L_{t}^{2} L_{x}^{\frac{2 d}{d-3}}}^{\frac{4}{d+1}}} \\
& \cdot\left(\left\||\nabla|^{\frac{1}{2}} v\right\|_{L_{t, x}^{\frac{2(d-1)}{d-1}}}\left\|\tilde{\psi}_{k} W^{-1}\right\|_{L_{x}^{\infty}}+\left\||\nabla|^{\frac{1}{2}}\left(\tilde{\psi}_{k} W^{-1}\right)\right\|_{L_{x}^{2 d}}\|v\|_{L_{t}^{\frac{2(d+1)}{d-1}}} \frac{\frac{2 d(d+1)}{L_{x}^{2}-2 d-1}}{d^{2}}\right) \\
& \lesssim\left\|W^{p_{c}} \tilde{\psi}_{k}\right\|_{L_{x}^{q_{1}}}\left\|\tilde{\psi}_{k} W^{-1}\right\|_{L_{x}^{\infty}}^{\frac{4}{d+1}}\|v\|_{\dot{S}^{1}(I)}^{1+\frac{4}{d+1}}\left(\left\|\tilde{\psi}_{k} W^{-1}\right\|_{L_{x}^{\infty}}+\left\||\nabla|^{\frac{1}{2}}\left(\tilde{\psi}_{k} W^{-1}\right)\right\|_{L_{x}^{2 d}}\right) \\
& \lesssim 2^{k\left(\frac{d}{q_{1}}-d-2+\frac{4(d-2)}{d+1}+d-2\right)}\|v\|_{\dot{S}^{1}(I)}^{1+\frac{4}{d+1}} \\
& \lesssim 2^{-\frac{6 k}{d+1}}\|v\|_{\dot{S}^{1}(I)}^{1+\frac{4}{d+1}} \text {. }
\end{aligned}
$$

Summing in $k$ we obtain

$$
\text { (4.9) } \lesssim\|v\|_{\dot{S}^{1}(I)}^{\frac{d+5}{d+1}}
$$


Collecting the estimates (4.10), (4.11) we obtain

$$
\left\||\nabla|^{\frac{1}{2}}\left(W^{p_{c}} J\left(\frac{v}{W}\right)\right)\right\|_{L_{t, x}^{\frac{2(d+1)}{d+3}}} \lesssim\|v\|_{\dot{S}^{1}(I)}^{\frac{d+3}{d+1}}+\|v\|_{\dot{S}^{1}(I)}^{\frac{d+5}{d+1}} .
$$

This together with (4.5), (4.6) yields that

$$
\|R(v)\|_{\dot{N}^{1}(I)} \lesssim\|v\|_{\dot{S}^{1}(I)}^{\frac{d+3}{d+1}}+\|v\|_{\dot{S}^{1}(I)}^{p_{c}}
$$

Lemma 4.1 is proved.

Based on this estimate, we have the following gain of decay estimate.

Lemma 4.2 (Gain of decay). Let $w^{a}=W^{a}-W$. Then for sufficiently large $t$ we have

$$
\begin{aligned}
& \left\|R\left(h+w^{a}\right)(t)-R\left(w^{a}\right)(t)\right\|_{L_{x}^{\frac{2 d}{d+2}}} \lesssim\|h(t)\|_{\dot{H}_{x}^{1}}^{p_{c}}+\|h(t)\|_{\dot{H}_{x}^{1}} e^{-\left(p_{c}-1\right) e_{0} t} \\
& \left\|R\left(h+w^{a}\right)-R\left(w^{a}\right)\right\|_{\dot{N}^{1}([t, \infty))} \\
& \quad \lesssim\|h\|_{\dot{S}^{1}([t, \infty))}^{p_{c}}+\|h\|_{\dot{S}^{1}([t, \infty))}^{\frac{d+3}{d+1}}+\|h\|_{\dot{S}^{1}([t, \infty))} e^{-\left(p_{c}-1\right) e_{0} t}
\end{aligned}
$$

Proof. Noting from Corollary 3.4 that $\left|w^{a}(t, x)\right| \leq \frac{1}{4} W(x)$, we have $w^{a}+W>\frac{1}{2} W$. We can write

$$
\begin{aligned}
& R\left(h+w^{a}\right)-R\left(w^{a}\right) \\
& =\left|h+w^{a}+W\right|^{p_{c}-1}\left(h+w^{a}+W\right)-\left|w^{a}+W\right|^{p_{c}-1}\left(w^{a}+W\right)-p_{c} W^{p_{c}-1} h \\
& =\left|h+w^{a}+W\right|^{p_{c}-1}\left(h+w^{a}+W\right)-\left|w^{a}+W\right|^{p_{c}-1}\left(w^{a}+W\right)-p_{c}\left|w^{a}+W\right|^{p_{c}-1} h \\
& \quad+p_{c} h\left(\left|w^{a}+W\right|^{p_{c}-1}-W^{p_{c}-1}\right) \\
& =\left|w^{a}+W\right|^{p_{c}} J\left(\frac{h}{w^{a}+W}\right)+p_{c} h\left(\left|w^{a}+W\right|^{p_{c}-1}-W^{p_{c}-1}\right)+|h|^{p_{c}-1} h,
\end{aligned}
$$

where $J(\cdot)$ is defined in (4.3). We first prove (4.13). Noting by (4.4) $|J(s)| \lesssim|s|^{p_{c}}$ and using Corollary 3.4 we have

$$
\begin{aligned}
\left\|R\left(h+w^{a}\right)(t)-R\left(w^{a}\right)(t)\right\|_{L_{x}^{\frac{2 d}{d+2}}} & \lesssim\left\|\left.||\right|^{p_{c}}\right\|_{L_{x}^{\frac{2 d}{d+2}}}+\left\|h\left|w^{a}\right|^{p_{c}-1}\right\|_{L_{x}^{\frac{2 d}{d+2}}} \\
& \lesssim\|h(t)\|_{\dot{H}_{x}^{1}}^{p_{c}}+\|h(t)\|_{\dot{H}_{x}^{1}}\left\|w^{a}(t)\right\|_{L_{x}^{\frac{2 d}{d-2}}}^{p^{\frac{2 d}{d-2}}} \\
& \lesssim\|h(t)\|_{\dot{H}_{x}^{1}}^{p_{c}}+\|h(t)\|_{\dot{H}_{x}^{1}} e^{-\left(p_{c}-1\right) e_{0} t} .
\end{aligned}
$$

Next we prove (4.14). By the triangle inequality we have

$$
\begin{aligned}
& \left\|R\left(h+w^{a}\right)-R\left(w^{a}\right)\right\|_{\dot{N}^{1}([t, \infty))} \\
\lesssim & \left\|\left.\nabla\right|^{\frac{1}{2}}\left(\left|w^{a}+W\right|^{p_{c}} J\left(\frac{h}{w^{a}+W}\right)\right)\right\|_{L_{t, x}^{\frac{2(d+1)}{d+3}}} \\
& +\left\|h\left(\left|w^{a}+W\right|^{p_{c}-1}-W^{p_{c}-1}\right)\right\|_{L_{t}^{1} L_{x}^{2}}+\left\||\nabla|^{\frac{1}{2}}\left(|h|^{p_{c}-1} h\right)\right\|_{L_{t, x}^{\frac{2(d+1)}{d+3}}}
\end{aligned}
$$

The third term on the RHS has been treated (see (4.61)), so we have

$$
\left\||\nabla|^{\frac{1}{2}}\left(|h|^{p_{c}-1} h\right)\right\|_{L_{t, x}^{\frac{2(d+1)}{d+3}}} \lesssim\|h\|_{S^{1}([t, \infty))}^{p_{c}}
$$


For the second term, noting that for $d \geq 6$,

$$
|| w^{a}+\left.W\right|^{p_{c}-1}-\left.W^{p_{c}-1}|\leq| w^{a}\right|^{p_{c}-1},
$$

we estimate, by using Corollary 3.4 ,

$$
\begin{aligned}
\left\|h\left(\left|w^{a}+W\right|^{p_{c}-1}-W^{p_{c}-1}\right)\right\|_{L_{s}^{1} L_{x}^{2}} & \lesssim\|h\|_{L_{s}^{2} L_{x}^{\frac{2 d}{d-3}}}\left\|\left|w^{a}\right|^{p_{c}-1}\right\|_{L_{s}^{2} L_{x}^{\frac{2 d}{3}}} \\
& \lesssim e^{-\left(p_{c}-1\right) e_{0} t}\|h\|_{\dot{S}^{1}([t, \infty))} .
\end{aligned}
$$

This is good for us. To estimate the first term, we borrow the proof of (4.12), which is still valid if we replace $W$ by another function having the same decay. Indeed, by checking the proof of (4.12), we easily find that all we need is the following:

$$
\begin{array}{r}
\left\||\nabla|^{\frac{1}{2}}\left(W^{\alpha} \psi_{k}\right)\right\|_{p} \lesssim 2^{k\left(\frac{d}{p}-\alpha(d-2)-\frac{1}{2}\right)}, \quad \forall \alpha>0, \\
\left\|W^{\beta} \psi_{k}\right\|_{p} \lesssim 2^{k\left(\frac{d}{p}-\beta(d-2)\right)}, \quad \forall \beta \in \mathbb{R},
\end{array}
$$

with the same estimates holding for $\tilde{\psi}_{k}$. Using Corollary 3.4, we verify that (4.17), (4.18) hold if we replace $W$ by $w^{a}+W$. Thus, the same proof in proving (4.12) establishes that

$$
\left\||\nabla|^{\frac{1}{2}}\left(\left(w^{a}+W\right)^{p_{c}} J\left(\frac{v}{w^{a}+W}\right)\right)\right\|_{L_{t, x}^{\frac{2(d+1)}{d+3}}} \lesssim\|h\|_{\dot{S}^{1}([t, \infty))}^{\frac{d+3}{d+1}}+\|h\|_{\dot{S}^{1}([t, \infty))}^{\frac{d+5}{d+1}} .
$$

Collecting the estimates (4.15), (4.16), (4.19), we obtain (4.14).

\section{Classification of the solution}

Our purpose in this section is to prove Theorem 1.4. Following the argument in [4, the key step is to establish the following.

Theorem 5.1. Let $\gamma_{0}>0$. Assume $u$ is the solution of the equation in (1.1) satisfying $E\left(u_{0}, u_{1}\right)=E(W, 0)$ and

$$
\|\nabla(u(t)-W)\|_{2}+\left\|\partial_{t} u(t)\right\|_{2} \leq C e^{-\gamma_{0} t}, \quad \forall t \geq 0 .
$$

Then there exists a unique $a \in \mathbb{R}$ such that

$$
u=W^{a} .
$$

As a corollary of Theorem 5.1, we see that modulo time translation, all the $\left\{W^{a}, a>0\right\}$ (also $\left\{W^{a}, a<0\right\}$ ) are the same.

Corollary 5.2. For any $a \neq 0$, there exists $T_{a} \in \mathbb{R}$ such that

$$
\begin{cases}W^{a}(t)=W^{+}\left(t+T_{a}\right), & \text { if } a>0, \\ W^{a}(t)=W^{-}\left(t+T_{a}\right), & \text { if } a<0 .\end{cases}
$$

We now prove Theorem 5.1. The strategy is the following: we first prove that there exists $a \in \mathbb{R}$ such that $\left\|\nabla\left(u(t)-W^{a}(t)\right)\right\|_{2}+\left\|\partial_{t}\left(u(t)-W^{a}(t)\right)\right\|_{2}$ has enough decay; then using the decay estimate we show that $u(t)-W^{a}(t)$ is actually identically zero. To this end, we have to input the condition (5.1) and upgrade it to the desired decay estimate. At this point, we need the following crucial result from [4. 
Lemma 5.3. 2 Let $t_{0} \geq 0$. Let $h$ be the solution to the equation

$$
\partial_{t t} h+\mathcal{L} h=\varepsilon, t \geq t_{0},
$$

where $h \in C^{0}\left(\left[t_{0}, \infty\right) ; \dot{H}_{x}^{1}\right), \partial_{t} h \in C^{0}\left(\left[t_{0}, \infty\right) ; L_{x}^{2}\right), \varepsilon \in \dot{N}^{1}\left(\left[t_{0}, \infty\right)\right)$. Assume for some constant $c_{0}, c_{1}$ such that $0<c_{0}<c_{1}$,

$$
\begin{aligned}
\|\nabla h(t)\|_{2}+\left\|\partial_{t} h(t)\right\|_{2} & \leq C e^{-c_{0} t}, \\
\|\varepsilon(t)\|_{L_{x}^{\frac{2 d}{d+2}}}+\|\varepsilon\|_{\dot{N}^{1}([t, \infty))} & \leq C e^{-c_{1} t} .
\end{aligned}
$$

Let $c_{1}^{-}$be an arbitrary number smaller than $c_{1}$. Then the following statements hold true.

- If $c_{1}>e_{0}$, there exists $A \in \mathbb{R}$ such that

$\left\|\partial_{t}\left(h(t)-A e^{-e_{0} t} \mathcal{Y}\right)\right\|_{2}+\left\|\nabla\left(h(t)-A e^{-e_{0} t} \mathcal{Y}\right)\right\|_{2}+\left\|h-A e^{-e_{0} t}\right\|_{\dot{S}^{1}([t, \infty))} \leq C e^{-c_{1}^{-} t}$

- If $c_{1} \leq e_{0}$,

$$
\|\nabla h(t)\|_{2}+\left\|\partial_{t} h(t)\right\|_{2}+\|h\|_{\dot{S}^{1}([t, \infty))} \leq C e^{-c_{1}^{-} t} .
$$

We give the proof of Theorem 5.1 .

Proof of Theorem 5.1. We divide the proof into three steps.

Step 1. Let $v=u-W$. Then condition (5.1) gives that

$$
\|\nabla v(t)\|_{2}+\left\|\partial_{t} v(t)\right\|_{2} \leq C e^{-\gamma_{0} t} .
$$

WOLOG, we can assume $\gamma_{0}<e_{0}$. We first show that this decay rate can be upgraded to $e^{-e_{0}^{-} t}$. More precisely, we will prove that

$$
\left\{\begin{array}{l}
\|\nabla v(t)\|_{2}+\left\|\partial_{t} v(t)\right\|_{2} \leq C e^{-e_{0}^{-} t}, \\
\|R(v)(t)\|_{L_{x}^{\frac{2 d}{d+2}}}+\|R(v)\|_{\dot{N}^{1}([t, \infty))} \leq C e^{-\frac{d+3}{d+1} e_{0}^{-} t},
\end{array}\right.
$$

and that there exists $a \in \mathbb{R}$ such that $\forall \eta>0$,

$$
\begin{aligned}
\left\|\nabla\left(v(t)-a e^{-e_{0} t} \mathcal{Y}\right)\right\|_{2} & +\left\|\partial_{t}\left(v(t)-a e^{-e_{0} t} \mathcal{Y}\right)\right\|_{2} \\
& +\left\|v-a e^{-e_{0} s} \mathcal{Y}\right\|_{\dot{S}^{1}([t, \infty))} \leq C_{\eta} e^{-\left(\frac{d+3}{d+1}-\eta\right) e_{0} t} .
\end{aligned}
$$

Note that (5.10) is a consequence of (5.9). Indeed assume that (5.9) is true. Then since $v$ satisfies the equation

$$
\partial_{t t} v+\mathcal{L} v=R(v)
$$

applying Lemma 5.3 with $h=v, \varepsilon=R(v)$ and $c_{0}=e_{0}^{-}, c_{1}=\frac{d+3}{d+1} e_{0}^{-}$, we obtain (5.10). It remains to show (5.9) by using the condition (5.8). To begin with we show that (5.8) implies that

$$
\|v\|_{\dot{S}^{1}([t, \infty))} \leq C e^{-\gamma_{0} t}
$$

\footnotetext{
${ }^{2}$ The presentation of Lemma 5.3 is slightly different from Proposition 5.7 in [4]. Here we use a weaker condition (5.4), (5.5) to yield stronger conclusions (5.6), (5.7). However, one can easily find that this change is harmless once we apply the Strichartz estimate and repeat the same argument in establishing Proposition 5.7 in [4].
} 
Let $s \geq t$. Let $\tau>0$ be a small constant to be chosen later. Using the Strichartz estimate on $[s, s+\tau]$ and Lemma 4.1 we have

$$
\begin{aligned}
\|v\|_{\dot{S}^{1}([s, s+\tau])} \lesssim & \|\nabla v(s)\|_{2}+\left\|v_{t}(s)\right\|_{2}+\left\|W^{p_{c}-1} v\right\|_{L_{t}^{1} L_{x}^{2}\left([s, s+\tau] \times \mathbb{R}^{d}\right)}+\|R(v)\|_{\dot{N}^{1}([s, s+\tau])} \\
\lesssim & e^{-\gamma_{0} s}+\tau\|v\|_{L_{t}^{\infty} \dot{H}_{x}^{1}\left([s, s+\tau] \times \mathbb{R}^{d}\right)}\left\|W^{p_{c}-1}\right\|_{L_{x}^{d}}+\|v\|_{\dot{S}^{1}([s, s+\tau])}^{\frac{d+3}{d+1}} \\
& +\|v\|_{\dot{S}_{c}^{1}([s, s+\tau])}^{p_{1}} \\
\lesssim & e^{-\gamma_{0} s}+\tau\|v\|_{\dot{S}^{1}([s, s+\tau])}+\|v\|_{\dot{S}^{1}([s, s+\tau])}^{p_{c}}+\|v\|_{\dot{S}^{1}([s, s+\tau])}^{\frac{d+3}{d+1}}
\end{aligned}
$$

Taking $\tau$ small enough, by a continuity argument we have

$$
\|v\|_{\dot{S}^{1}([s, s+\tau])} \leq C e^{-\gamma_{0} s} .
$$

By the triangle inequality we obtain

$$
\begin{aligned}
\|v\|_{\dot{S}^{1}([t, \infty))} & \leq \sum_{j \geq 0}\|v\|_{\dot{S}^{1}([t+\tau j, t+\tau(j+1)])} \\
& \leq C \sum_{j \geq 0} e^{-\gamma_{0}(t+\tau j)} \\
& \lesssim e^{-\gamma_{0} t}
\end{aligned}
$$

Lemma 4.1 yields that

$$
\|R(v)(t)\|_{L_{x}^{\frac{2 d}{d+2}}} \lesssim e^{-\frac{d+3}{d+1} \gamma_{0} t}, \quad\|R(v)\|_{\dot{N}^{1}([t, \infty))} \leq e^{-\frac{d+3}{d+1} \gamma_{0} t} .
$$

Now we can apply Lemma 5.3 to obtain

$$
\|\nabla v(t)\|_{2}+\left\|\partial_{t} v(t)\right\|_{2}+\|v\|_{\dot{S}^{1}([t, \infty))} \leq C\left(e^{-e_{0}^{-} t}+e^{-\frac{d+2}{d+1} \gamma_{0} t}\right) .
$$

If $\frac{d+2}{d+1} \gamma_{0} \geq e_{0},(5.9)$ is proved. Otherwise, we are in the same situation with $\gamma_{0}$ being replaced by $\frac{d+2}{d+1} \gamma_{0}$. Iterating this process finitely many times yields (5.9).

Step 2. In this step we prove that $u(t)-W^{a}(t)$ decays arbitrarily fast. We prove that $\forall m>0$, there exists $t_{m}>0$ such that

$$
\left\|u-W^{a}\right\|_{\dot{S}^{1}([t, \infty))} \leq e^{-m t}, t \geq t_{m} .
$$

To begin with, we show that (5.11) holds for $m=\frac{d+2}{d+1} e_{0}$. Indeed, by the triangle inequality and recalling that $v=u-W$, we estimate

$$
\begin{aligned}
& \left\|u-W^{a}\right\|_{\dot{S}^{1}([t, \infty))} \\
\leq & \left\|v-a e^{-e_{0} s} \mathcal{Y}\right\|_{\dot{S}^{1}([t, \infty))}+\left\|w^{a}-v_{k_{0}}\right\|_{\dot{S}^{1}([t, \infty))}+\left\|v_{k_{0}}-a e^{-e_{0} s} \mathcal{Y}\right\|_{\dot{S}^{1}([t, \infty))} .
\end{aligned}
$$

For the first term, we use (5.10) to estimate

$$
\left\|v-a e^{-e_{0} s} \mathcal{Y}\right\|_{\dot{S}^{1}([t, \infty))} \leq \frac{1}{3} e^{-\frac{d+2}{d+1} e_{0} t} .
$$

For the last two terms, we use the definition of $v_{k_{0}}$ and Corollary 3.4 to get

$$
\begin{aligned}
\left\|w^{a}-v_{k_{0}}\right\|_{\dot{S}^{1}([t, \infty))} & \leq e^{-k_{0} e_{0} t} \leq \frac{1}{3} e^{-\frac{d+2}{d+1} e_{0} t} \\
\left\|v_{k_{0}}-a e^{-e_{0} s} \mathcal{Y}\right\|_{\dot{S}^{1}([t, \infty))} & \leq\left\|\sum_{2 \leq j \leq k_{0}} e^{-j e_{0} t} \Phi_{j}\right\|_{\dot{S}^{1}([t, \infty))} \\
& \lesssim e^{-2 e_{0} t} \leq \frac{1}{3} e^{-\frac{d+2}{d+1} e_{0} t}
\end{aligned}
$$


Collecting these estimates together we obtain

$$
\left\|u-W^{a}\right\|_{\dot{S}^{1}([t, \infty))} \leq e^{-\frac{d+2}{d+1} e_{0} t} .
$$

Now supposing that (5.11) holds for some $m_{1} \geq \frac{d+2}{d+1} e_{0}$, we show that (5.11) holds for $m=m_{1}+\frac{1}{d+1} e_{0}$. This will yield (5.11) by iteration.

Write $h=u-W^{a}$. Then $h$ satisfies

$$
\partial_{t t} h+\mathcal{L} h=R\left(h+w^{a}\right)-R\left(w^{a}\right) .
$$

Since by Lemma 4.2, we have

$$
\begin{aligned}
\left\|R\left(h+w^{a}\right)(t)-R\left(w^{a}\right)(t)\right\|_{L_{x}^{\frac{2 d}{d+2}}} \lesssim & \|h(t)\|_{\dot{H}_{x}^{1}}^{p_{c}}+\|h(t)\|_{\dot{H}_{x}^{1}} e^{-\left(p_{c}-1\right) e_{0} t} \\
\lesssim & e^{-\left(m_{1}+\left(p_{c}-1\right) e_{0}\right) t} \\
\left\|R\left(h+w^{a}\right)-R\left(w^{a}\right)\right\|_{\dot{N}^{1}([t, \infty))} \lesssim & \|h\|_{\dot{S}^{1}([t, \infty))}^{p_{c}}+e^{-\left(p_{c}-1\right) e_{0} t}\|h\|_{\dot{S}^{1}([t, \infty))} \\
& +\|h\|_{\dot{S}^{1}([t, \infty))}^{\frac{d+3}{d+1}} \\
\lesssim & e^{-\left(m_{1}+\frac{2}{d+1} e_{0}\right) t},
\end{aligned}
$$

applying Lemma 5.3 again gives us

$$
\|h\|_{\dot{S}^{1}([t, \infty))} \leq e^{-\left(m_{1}+\frac{1}{d+1} e_{0}\right) t} .
$$

Step 3. Now we show that there exists $m>0$ such that $h(t)=0$ for all $t \geq t_{m}$ by using the decay estimate (5.11).

First we note that $h$ satisfies the equation

$$
\partial_{t t} h-\Delta h=p_{c} W^{p_{c}-1} h+R\left(w^{a}+h\right)-R\left(w^{a}\right),
$$

and the following Duhamel's formula:

$$
h(t)=-\int_{t}^{\infty} \frac{\sin ((t-s) \sqrt{-\Delta})}{\sqrt{-\Delta}}\left(p_{c} W^{p_{c}-1} h+R\left(W^{a}+h\right)-R\left(W^{a}\right)\right)(s) d s .
$$

Applying the Strichartz estimate on $[t, \infty)$, we obtain

$$
\|h\|_{\dot{S}^{1}([t, \infty))} \leq C\left(\left\|W^{p_{c}-1} h\right\|_{L_{s}^{1} L_{x}^{2}\left([t, \infty) \times \mathbb{R}^{d}\right)}+\left\|R\left(h+w^{a}\right)-R\left(w^{a}\right)\right\|_{\dot{N}^{1}([t, \infty))}\right) .
$$

Denote $\|h\|_{\Sigma_{t}}:=\sup _{s \geq t} e^{m s}\|h\|_{\dot{S}^{1}([s, \infty))}$. Then for $\eta>0$ small enough we have

$$
\begin{aligned}
\left\|W^{p_{c}-1} h\right\|_{L_{s}^{1} L_{x}^{2}\left([t, \infty) \times \mathbb{R}^{d}\right)} & \lesssim \sum_{j \geq 0}\left\|W^{p_{c}-1} h\right\|_{L_{s}^{1} L_{x}^{2}\left([t+\eta j, t+\eta(j+1)] \times \mathbb{R}^{d}\right)} \\
& \leq \eta\left\|W^{p_{c}-1}\right\|_{L_{x}^{d}}\|h\|_{L_{s}^{\infty} L_{x}^{\frac{2 d}{d-2}}\left([t+\eta j, t+\eta(j+1)] \times \mathbb{R}^{d}\right)} \\
& \lesssim \sum_{j \geq 0} \eta\|h\|_{\dot{S}^{1}([t+\eta j, t+(j+1) \eta])} \\
& \lesssim \sum_{j \geq 0} \eta e^{-m(t+\eta j)}\|h\|_{\Sigma_{t_{m}}} \\
& \lesssim e^{-m t}\|h\|_{\Sigma_{t_{m}}} \frac{\eta}{1-e^{-\eta m}} \\
& \lesssim \frac{2}{m} e^{-m t}\|h\|_{\Sigma_{t_{m}}} .
\end{aligned}
$$


From Lemma 4.2 we get

$$
\left\|R\left(w^{a}+h\right)-R\left(w^{a}\right)\right\|_{\dot{N}^{1}([t, \infty))} \leq \frac{1}{10 C} e^{-m t}\|h\|_{\Sigma_{t_{m}}} .
$$

Combining these two estimates, we get

$$
\|h\|_{\Sigma_{t_{m}}} \leq \frac{1}{2}\|h\|_{\Sigma_{t_{m}}}
$$

which implies that $h(t)=0$ on $\left[t_{m}, \infty\right)$. Recalling that $h(t)=u(t)-W^{a}(t)$, we obtain $u(t)=W^{a}(t)$ on $\left[t_{m}, \infty\right)$. Therefore $u \equiv W^{a}$ by the uniqueness of solutions to (1.1). The proposition is proved and we have Theorem 5.1.

Proof of Corollary 5.2. The proof is almost the same as Corollary 6.5 in [4. Let $a \neq 0$ and $T_{a}$ be such that $|a| e^{-e_{0} T_{a}}=1$. By (3.7) we have

$$
\left\|W^{a}\left(t+T_{a}\right)-W \mp e^{-e_{0} t} \mathcal{Y}\right\|_{H^{m, m}} \lesssim e^{-\frac{3}{2} e_{0} t} .
$$

Moreover $W^{a}\left(\cdot+T_{a}\right)$ satisfies the assumption in Theorem 5.1, thus there exists $a^{\prime}$ such that $W^{a}\left(\cdot+T_{a}\right)=W^{a^{\prime}}$. By (5.12), $a^{\prime}=1$ if $a>0$ and $a^{\prime}=-1$ if $a<0$. Corollary 5.2 is proved.

Finally, we give the proof of the main theorem, Theorem 1.4 .

Proof of Theorem 1.4. We first note that (b) is just the variational characterization of $W$. More precisely we have

Theorem $5.4([1,23])$. Let $c(d)$ denote the sharp constant in the Sobolev embedding

$$
\|f\|_{\frac{2 d}{d-2}} \leq c(d)\|\nabla f\|_{2} \text {. }
$$

Then the equality holds iff $f$ is $W$ up to symmetries. More precisely, there exists $\lambda_{0}>0, x_{0} \in \mathbb{R}^{d}, \delta_{0} \in\{-1,+1\}$, such that

$$
f(x)=\delta_{0} \lambda_{0}^{-\frac{d-2}{2}} W\left(\frac{x-x_{0}}{\lambda_{0}}\right) .
$$

In particular, if $\left(u_{0}, u_{1}\right) \in \dot{H}_{x}^{1} \times L_{x}^{2}$ satisfies

$$
E\left(u_{0}, u_{1}\right)=E(W, 0),\left\|\nabla u_{0}\right\|_{2}=\|\nabla W\|_{2},
$$

then $\left(u_{0}, u_{1}\right)=(W, 0)$ up to symmetries; hence the corresponding solution u coincides with $W$ up to symmetries.

It remains for us to show (a), (c). We first prove (a). Let $u$ be the maximallifespan solution of (1.1) on $I$ satisfying $E\left(u_{0}, u_{1}\right)=E(W, 0),\left\|\nabla u_{0}\right\|_{2}<\|\nabla W\|_{2}$. Then by Proposition 1.5, we have $I=\mathbb{R}$. Assume that $u$ blows up forward in time. Applying Proposition[1.5]again, we conclude that there exist $x_{0} \in \mathbb{R}^{d}, \mu_{0}, \gamma_{0}, C>0$ such that

$$
\left\|\nabla\left(u(t)-W_{\left[\mu_{0}, x_{0}\right]}\right)\right\|_{2}+\left\|\partial_{t} u(t)\right\|_{2} \leq C e^{-\gamma_{0} t},
$$

where $W_{\left[\mu_{0}, x_{0}\right]}=\mu_{0}^{-\frac{d-2}{2}} W\left(\frac{x+x_{0}}{\mu_{0}}\right)$. This implies

$$
\left\|\nabla\left(u_{\left[\mu_{0}^{-1},-\mu_{0}^{-1} x_{0}\right]}(t)-W\right)\right\|_{2}+\left\|\partial_{t}\left(u_{\left[\mu_{0}^{-1},-\mu_{0}^{-1} x_{0}\right]}(t)\right)\right\|_{2} \leq e^{-\gamma_{0} \mu_{0} t},
$$

where

$$
u_{\left[\mu_{0}^{-1},-\mu_{0}^{-1} x_{0}\right]}(t, x)=\mu_{0}^{\frac{d-2}{2}} u\left(\mu_{0} t, \mu_{0} x-x_{0}\right)
$$

is also a solution of the equation (1.1). By Theorem 5.1 with $\gamma_{0}$ now replaced by $\gamma_{0} \mu_{0}$, we conclude that there exists $a<0$ such that $u_{\left[\mu_{0}^{-1},-\mu_{0}^{-1} x_{0}\right]}=W^{a}$. 
Using Corollary 5.2, we get

$$
u(t, x)=\mu_{0}^{-\frac{d-2}{2}} W^{-}\left(\mu_{0}^{-1} t+T_{a}, \mu_{0}^{-1}\left(x+x_{0}\right)\right) .
$$

This shows that $u=W^{-}$up to symmetries. The proof of (c) is similar, so we omit it. This ends the proof of Theorem 1.4 .

\section{ACKNOWLEDGEMENTS}

Both authors were supported by the National Science Foundation under agreement No. DMS-0635607. The first author was supported by NSF Grant-0908032 and a start-up funding from the University of Iowa. The second author was supported by an Alfred P. Sloan fellowship and also a start-up funding from the University of Iowa.

\section{REFERENCES}

1. T. Aubin, Équations différentielles non linéaires et problème de Yamabe concernant la courbure scalaire. J. Math. Pures Appl. (9), 55(3): 269-296, 1976. MR0431287 (55:4288)

2. A. Bulut, M. Czubak, D. Li, N. Pavlović and X. Zhang, Stability and unconditional uniqueness of solutions for energy critical wave equations in high dimensions, submitted.

3. T. Duyckaerts, F. Merle, Dynamics of threshold solutions for energy-critical NLS, Geom. Funct. Anal. 18 (2009), 1787-1840. MR2491692 (2010e:35258)

4. T. Duyckaerts, F. Merle, Dynamics of threshold solutions for energy-critical wave equation, Int. Math. Res. Pap. 2007, no. 4, ID rpn002, 67 pp. (2008). MR2416069

5. J. Ginibre, A. Soffer, G. Velo, The global Cauchy problem for the critical nolinear wave equation, J. Funct. Anal. 110 (1992), 96-130. MR.1190421 (94d:35105)

6. J. Ginibre, G. Velo, Generalized Strichartz inequalities for the wave equation. J. Funct. Anal., 133(1): 50-68, 1995. MR.1351643 (97a:46047)

7. M. Grillakis, Regularity and asymptotic behavior of the wave equation with a critical nonlinearity, Ann. of Math. (2) 132 (1990), 485-509. MR.1078267 (92c:35080)

8. M. Grillakis, Regularity for the wave equation with a critical nonlinearity, Comm. Pure. Appl. Math. 45 (1992), 749-774. MR1162370 (93e:35073)

9. L. Kapitanski, Global and unique weak solutions of nonlinear wave equations, Math. Res. Lett., 1 (1994), no. 2, 211-223. MR.1266760 (95f:35158)

10. M. Keel and T. Tao, Endpoint Strichartz Estimates, Amer. J. Math. 120 (1998), 955-980. MR.1646048 (2000d:35018)

11. C. Kenig and F. Merle, Global well-posedness, scattering and blow-up for the energy-critical focusing non-linear wave equation, Acta Mathematica 201 (2008), 147-212. MR2461508

12. C. Kenig, G. Ponce, L. Vega, Well-posedness and scattering results for the generalized Korteweg-de Vries equation via the contraction principle. Comm. Pure. Appl. Math. 46(4): 527-620. MR 1211741 (94h:35229)

13. R. Killip, M. Visan, Nonlinear Schrödinger equations at critical regularity, Clay lecture notes.

14. R. Killip, M. Visan, The focusing energy-critical nonlinear Schrödinger equation in dimensions five and higher, Amer. J. Math. 132 (2010), 361-424. MR2654778

15. D. Li, X. Zhang, Dynamics for the energy critical nonlinear Schrödinger equation in high dimensions. J. Funct. Anal. 256 (2009), 1928-1961. MR2498565 (2010b:35436)

16. H. Lindblad, C. Sogge, On existence and scattering with minimal regularity for semilinear wave equations. J. Funct. Anal. 130(2): 357-426, 1995. MR1335386 (96i:35087)

17. J. Shatah and M. Struwe, Regularity results for nonlinear wave equations, Ann. of Math. (2) 138 (1993), 503-518. MR:1247991 (95f:35164)

18. J. Shatah and M. Struwe, Well-posedness in the energy space for semilinear wave equations with critical growth, Internat. Math. Res. Notices 7 (1994), 303-309. MR 1283026 (95e:35132)

19. J. Shatah and M. Struwe, Geometric wave equations, Courant Lecture Notes in Mathematics, 2(1998). MR 1674843(2000i:35135)

20. C. Sogge, Lectures on nonlinear wave equations, Monographs in Analysis II, International Press, 1995. MR 1715192(2000g:35153) 
21. M. Struwe, Globally regular solutions to the $u^{5}$ Klein-Gordon equation, Ann. Scuola Norm. Sup. Pisa Cl. Sci. 15 (1988), 495-513. MR1015805 (90j:35142)

22. T. Tao, M. Visan, Stability of energy-critical nonlinear Schrödinger equations in high dimensions, Electron. J. Diff. Eqns. 118 (2005), 1-28. MR2174550 (2006e:35307)

23. G. Talenti, Best constant in Sobolev inequality, Ann. Mat. Pura. Appl. (4), 110: 353-372, 1976. MR0463908 (57:3846)

Department of Mathematics, University of Iowa, Maclean Hall, Iowa City, Iowa 52242

E-mail address: mpdongLi@gmail.com

Department of Mathematics, University of Iowa, Maclean Hall, Iowa City, Iowa 52242 - And - Chinese Academy of Sciences, Beijing, People's Republic of China

E-mail address: zh.xiaoyi@gmail.com 